\title{
Norois
}

Environnement, aménagement, société

$246 \mid 2018$

Eaux, marais, lac, étang, pêche

\section{Note de recherche - La recherche en géographie de la pêche : thèses et publications dans la revue Norois}

Research into the geography of fishing: PhD theses and Norois journal papers

\section{Yvanne Bouvet}

\section{(2) OpenEdition}

Journals

Édition électronique

URL : http://journals.openedition.org/norois/6384

DOI : ERREUR PDO dans /localdata/www-bin/Core/Core/Db/Db.class.php L.34 : SQLSTATE[HY000]

[2006] MySQL server has gone away

ISBN : 978-2-7535-7539-4

ISSN : $1760-8546$

Éditeur

Presses universitaires de Rennes

Édition imprimée

Date de publication : 6 juin 2018

Pagination : 93-105

ISBN : $978-2-7535-7537-0$

ISSN : 0029-182X

Référence électronique

Yvanne Bouvet, « Note de recherche - La recherche en géographie de la pêche : thèses et publications dans la revue Norois », Norois [En ligne], 246 | 2018, mis en ligne le 20 juin 2020, consulté le 07 janvier 2021. URL : http://journals.openedition.org/norois/6384; DOI : https://doi.org/ERREUR PDO dans / localdata/www-bin/Core/Core/Db/Db.class.php L.34 : SQLSTATE[HY000] [2006] MySQL server has gone away 


Presses
Universitaires
de Rennes
www.pur-editions.fr

\title{
Note de recherche \\ La recherche en géographie de la pêche : thèses et publications dans la revue Norois
}

\author{
Research note - Research into the Geography of Fishing: \\ PhD Theses and Norois Journal Papers
}

Yvanne Bouvet

Université de Bretagne occidentale, UFR LSHS - Victor Segalen, EA 7462 Géoarchitecture, Territoires, urbanisation, biodiversité, environnement - 20 rue Duquesne, CS 93837, 29238 BrEST cedex 3 (France). (yvanne. bowvet@univ-brest.fr)

Résumé : Cette note de recherche propose d'avoir un regard rétrospectif sur les publications consacrées à la pêche maritime dans la revue des géographes de l'ouest, Norois et à travers les thèses de géographie soutenues ces dernières années. Cet «état de l'art » présente l'évolution quantitative année par année, en voyant les espaces étudiés, puis en synthétisant les grands courants de pensée à l'intérieur des articles ou travaux doctoraux en analysant le corpus de ces publications et recherches.

\begin{abstract}
This paper looks back on the scientific literature devoted to fishing activities in Norois - the academic journal of geographers in Western France - and in doctoral (PhD) theses defended in the field of geography in recent years. This state of the art presents year-byyear quantitative trends, taking into consideration the various areas studied, and summarizing the main schools of thought represented within this literature.
\end{abstract}

Mots clés : Géographie - pêche - épistémologie - bibliométrie

Keywords: geography - fishing - epistemology - bibliometrics

En se dotant d'une stratégie maritime nationale, poussée par l'Union européenne et par les régions littorales, la France va peut-être enfin reconnaitre l'intérêt des espaces maritimes qui la bordent, audelà des plages et des ports de commerce. La gestion intégrée des zones côtières (GIZC) contribue déjà à prendre en compte les activités marines et littorales dans une réflexion sur l'évolution des territoires maritimes. Les recherches en géographie ont contribué à cette prise de conscience même si celles sur les activités maritimes sont plutôt récentes à l'échelle de l'histoire des sciences, et notamment sur les activités halieutiques. Depuis 40 ans, la pêche maritime voit sa production stagner (Bouvet, 2014), ses espaces de productions et de distribution s'étendre à l'échelle mondiale, et la filière est fortement intégrée à un système globalisé (Noël, 2013). La géographie, en se préoccupant des impacts des activités humaines dans les océans, a toute sa place pour décrire et analyser les espaces, les enjeux, les 
acteurs mobilisés, et mettre au jour les évolutions et changements des espaces de la pêche. Une activité halieutique peut être étudiée à différentes échelles, du local au global, à travers des éléments émanant autant de l'écosystème que du socio-système, et irrigant des territoires multiples.

La présente contribution vise à faire le point sur l'évolution et l'actualité des recherches sur ce sujet et propose un regard rétrospectif sur les publications consacrées à la pêche maritime dans la revue des géographes de l'Ouest, Norois, ainsi que sur les thèses de géographie soutenues ces dernières années.

Après une présentation de la place de la géographie dans l'étude scientifique de la pêche, ainsi que du corpus, nous aborderons l'évolution quantitative des travaux en géographie halieutique et les différents espaces étudiés. Enfin, les grandes problématiques et approches abordées dans ces publications et recherches seront présentées synthétiquement, en les replaçant dans un monde universitaire en pleine évolution.

\section{Place de la géographie HALIEUTIQUE ET PRÉSENTATION DU CORPUS ${ }^{1}$}

La connaissance scientifique de l'océan débute au milieu du XIX ${ }^{\mathrm{e}}$ siècle, grâce à des campagnes de recherche en mer, souvent portées par les sociétés de géographie européennes, alors qu'émerge la géographie moderne. Les explorations des Sociétés de géographie, de Paris, de Berlin, de Moscou, royales de Belgique ou de Londres, sont à visée scientifique, et économique, pour approfondir les connaissances du monde, et elles vont aussi concerner les océans. Si quelques géographes prennent part à ces expéditions comme E. Von Drygalski, aidant à compléter les cartes surtout dans les océans arctique et antarctique $^{2}$, d'autres encouragent les expéditions et contribuent à « universitariser» les connaissances, comme Ferdinand Von Richthofen (1833-1905) qui créa l'Institut d'océanographie et de géographie de Berlin en 1902 ou plus tard avec la chaire d'océanographie crée en 1945 à l'université de Leningrad,

1. Corpus présenté en fin d'article.

2. Erich Von Drygalski qui mena une expédition sur le Gauss autour des îles Kerguelen et dans l'océan Antarctique pour étudier la circulation océanique et les profondeurs sous-marines entre 1901 et 1903, puis rédigea 20 volumes sur ses découvertes en étant Professeur de géographie à l'université de Munich jusqu'en 1934. incombant à V. Ju. Vizé, physicien et explorateur de l'Arctique (1886-1954) (Bodéré et al., 1984).

Alors que depuis le milieu du XIX ${ }^{\mathrm{e}}$ siècle, des géographes se penchent sur le fonctionnement de la planète et son organisation, tel Karl Ritter (17791859) et Alexander Von Humboldt (1769-1859), on aurait pu supposer que la discipline s'intéresserait à la mer, dans une approche globale de la planète. Pourtant, au $\mathrm{Xx}^{\mathrm{e}}$ siècle, l'océanographie va s'éloigner de la géographie, s'associant à la biologie marine (en France, aux États-Unis, en Scandinavie, au Royaume-Uni), à la géophysique et à l'hydrologie, notamment à travers la cartographie des fonds qui est confiée en France, au Service hydrologique et océanographique de la Marine nationale ${ }^{3}$.

L'école géographique française de Paul Vidal de la Blache ne s'est guère intéressée à la mer, et c'est par la géomorphologie que les géographes l'aborderont, grâce à André Guilcher (1913-1993) qui a porté toute une génération de géographes vers la géomorphologie et l'hydrologie littorale et marine à l'Institut de géographie de Paris jusqu'en 1970, puis à Brest pour développer l'enseignement universitaire en géographie de la mer. Il encadrera 25 thèses d'état dont 18 sont consacrées à des problématiques littorales et marines qui ont installé l'étude des espaces maritimes et littoraux dans la géographie française; cinq parmi celles-ci sont en géographie humaine dont quatre sur la pêche (Carré, 1995).

Si l'océanographie ne s'est pas préoccupée des activités humaines maritimes, la géographie, comme l'économie et le droit, vont s'approprier ces thématiques. Le pionnier est Camille Vallaux (18701954), qui ouvre la géographie vers les contextes économiques, sociaux et politiques de la Bretagne tout d'abord, puis vers la mer et les océans à travers de nombreux travaux dont le magistral Géographie générale des mers publiée en $1933^{4}$. Vient ensuite la thèse posthume de Charles Robert-Muller ${ }^{5}$ parue

\footnotetext{
3. Les domaines d'expertises du SHOM sont notamment : la bathymétrie, la sédimentologie, l'hydrodynamique côtière, l'océanographie, l’ingénierie des systèmes d'acquisition à la mer, l'information géographique maritime et littorale. Le SHOM collecte et diffuse des données de référence dans ces domaines et fournit des services d'« intelligence de la donnée », qui aident les acteurs de la mer et du littoral à utiliser de manière optimale les données [http://www.shom.fr/le-shom/presentation-generale/les-missions/].

4. Vallaux C., 1933, Géographie générale des mers, Paris, Alcan, 796 p, parmi 83 écrits recensés à la Bibliothèque Nationale de France [http://data.bnf. fr/documents-by-rdt/11927495/70]).

5. Robert-Muller C., 1994, Pêche et pêcheurs de la Bretagne atlantique, Paris, thèse posthume de doctorat d'État, publiée cher Armand Colin et mis au point par LE LANNOU M., 616 p.
} 
en 1944 sur la pêche en Bretagne atlantique, qui suit celle de Louis Papy (1903-1990) plus générale sur la côte Atlantique de la Loire à la Gironde ${ }^{6}$, et l'orientation plus économique des enseignements d'Aimé Perpillou à Paris (1902-1976) sur la pêche et les transports.

Les travaux de François Doumenge (1926-2008) sur le Japon et la mer, avec l'ouvrage Géographie des mers publié en 1965, donne enfin une place à la mer pourvoyeuse de ressources alimentaires, et de systèmes de production bien particuliers, tout comme l'ouvrage de Jacques Besançon, Géographie des pêches. À la fin des années 1970, sous la direction d'André Guilcher, des thèses d'État émergent, liant pêche et études régionales. Ainsi, si en 1966, François Doumenge garde une approche encore vidalienne dans sa thèse sur L'homme dans le Pacifique Sud, on peut voir une rupture épistémologique se dessiner par une approche géosystémique de l'activité halieutique avec les thèses d'État de Jean Chaussade-Redon dans les provinces maritimes du Canada (1980), puis de François Carré en Écosse (1988), Gilbert David sur le Vanuatu (1991) et surtout de Jean-Pierre Corlay au Danemark (1993).

Cette fin du $\mathrm{xx}^{\mathrm{e}}$ siècle est celle d'une évolution de la demande sociétale et politique autour de la gestion des ressources maritimes, interrogeant toutes les sciences humaines et sociales pour dépasser les explications biologiques des crises de production. Ainsi, la géographie, mais aussi la sociologie, l'économie, le droit, l'histoire vont s'intéresser aux questions posées par les filières de production halieutique. La convention adoptée en 1982 à Montego Bay met fin à la Mare liberum et instaure la reconnaissance des zones économiques exclusives (ZEE) pour les états possédant un littoral maritime. La dimension territoriale donnée aux pêcheries oblige les pouvoirs publics à se poser la question de la gestion et du renouvellement des ressources marines « sauvages », où les géographes, les juristes, les économistes, voire les historiens et les sociologues vont se retrouver. La géographie halieutique, pour reprendre le titre de la thèse de Jean-Pierre Corlay (1993), se caractérise par une approche systémique et la rencontre entre un potentiel marin et une exploitation productrice de biens. Elle va s'affirmer, autour de quelques chercheurs, avec des démarches

6. PAPY L., 1942, La côte atlantique de la Loire à la Gironde, Bordeaux, 2 tomes, 830 p. Louis Papy est fondateur des Cahiers d'outre-mer. plus économistes pour certaines, plus sociales ou écologues pour d'autres, introduisant la notion de développement, de protection des milieux ou d'organisation des sociétés maritimes.

Pour rendre compte de la place de la pêche en géographie, nous avons choisi de retenir les thèses et les articles de Norois, qui traitent directement ou fortement de la pêche maritime professionnelle, soit autour de la présentation d'une filière, soit autour d'une approche régionale. Nous avons effectué une recherche automatique dans le fichier central des thèses françaises [www.theses.fr/] enrichie de requêtes dans la base HAL [https://tel.archivesouvertes.fr/] en ciblant la géographie, autour de deux mots-clés "pêche » et « halieutique », recoupant avec le fond de la BnF [http://catalogue.bnf.fr]. Nous avons ainsi recensé les travaux soutenus sur ce thème, au vu des titres des thèses, de leurs résumés, en enrichissant cette base de nos connaissances bibliographiques. Depuis 1980, ce sont une soixantaine de thèses de géographie (État, $3^{\text {e }}$ cycle puis nouveau régime) qui ont été consacrées à la pêche.

Puis nous nous sommes intéressés à Norois, « revue de géographie générale... traditionnellement inscrite dans la France de l'Ouest et les régions de l'Atlantique nord ${ }^{7}$ ", et qui depuis 1954, rassemble les géographes des universités de l'ouest de la France (Angers, Brest, Caen, Limoges, Le Mans, Nantes, Rennes, La Rochelle, Poitiers et Tours). Avec Norois, on peut retracer l'évolution de la recherche en géographie, particulièrement dans le champ maritime, notamment grâce aux quarante Chroniques océanographiques publiées pendant de 1954 à 1993 par André Guilcher. Une recherche en ligne ${ }^{8}$ a permis de faire apparaître les 430 articles contenant les mots «pêche » et « halieutique », depuis l'origine de la revue. Cependant, l'occurrence ne veut pas dire que l'article soit centré sur l'activité halieutique. Nous avons donc consulté chaque résumé d'article lorsqu'il était disponible ou consulté l'article concerné. Ainsi, nous avons conservé 75 articles centrés sur la pêche depuis plus de 60 ans, en ne tenant pas compte des notes, des chroniques et comptes rendus.

\footnotetext{
7. Voir le site de Norois : [http://norois.revues.org/].

8. La mise en ligne de tous les numéros de la revue facilite la recherche, [http://www.persee.fr/collection/norois].
} 


\section{Thèses et articles, Analyse QUANTITATIVE ET TERRAINS D'ÉTUDES}

La première thèse d'État sur la pêche est celle de Jean Chaussade-Redon présentée en 1980, suivie de trois thèses de troisième cycle en 1982 et 1983. C'est l'amorce de la soixantaine de thèses qui seront soutenues, avec une période de forte production entre 1986 et 1998 avec une moyenne de 2,4 thèses par an, et une pointe avec six thèses en 1993, dont une thèse d'État. Depuis les années 2000, la production est moins forte mais constante, avec 1,7 thèse en moyenne chaque année (figure 1). Cette production modeste est directement liée aux choix faits par les universités dans les profils de recrutement des professeurs d'université, et donc des priorités accordées par les laboratoires entre les différentes thématiques de la recherche en géographique de la mer, entre géomorphologie et gestion des espaces littoraux, géomatique et géographie économique.

Les régions qui retiennent l'attention des géographes halieutes sont en majorité à l'extérieur de l'espace français et même européen. Ainsi, la France ne concerne qu'un quart des sujets, et deux tiers des thèses sont consacrées à un espace hors Europe. C'est l'Afrique qui attire les doctorants, avec plus de quatre thèses sur dix qui lui sont consacrées, souvent menées par des chercheurs africains venant faire leur thèse dans les universités françaises. En France, ce sont les espaces atlantiques qui retiennent le plus l'attention, avec seulement trois sujets sur seize consacrés à la Méditerranée et à la Mer du Nord (figure 2).

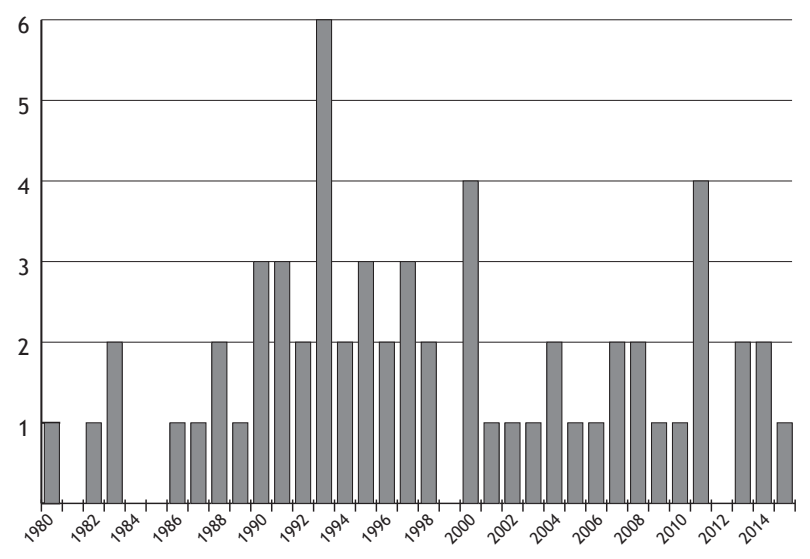

Figure 1 : Nombre de thèses consacrées à la pêche en géographie (sources : fichier central des thèses, HAL)

Number of geography theses devoted to fishing activities
C'est dans l'ouest de la France que sont réalisées le plus grand nombre de thèses, entre l'Université de Nantes (deux cinquièmes) et l'Université de Brest (un cinquième), là où se retrouvent les porteurs de la géographie de la mer en France. Si André Guilcher a encadré cinq thèses sur la pêche, Jean ChaussadeRedon en a encadré douze (soit plus d'un cinquième des thèses sur le sujet) et Jean-Pierre Corlay, six. Le départ de ces géographes halieutes n'a pas permis de maintenir le vivier des géographes halieutes dans les universités de l'Ouest. Les universités parisiennes sont moins présentes sur cette thématique, avec seulement sept thèses, alors que Bordeaux et Montpellier offrent régulièrement des études doctorales en géographie halieutique. Depuis la fin de la décennie 2000, on voit aussi d'autres universités accompagner des études doctorales sur cette thématique, souvent couplée avec une approche de géographie tropicale (Claquin, 2008; Ndeye, 2009; Ekouala, 2013).

Les publications sur la pêche dans Norois sont plus précoces (tableau 1). Si dans les premières années de publication il y en a peu, on constate un engouement à partir de la décennie 1970 avec 179 articles où se trouve le mot «pêche » dont 24 articles centrés sur le thème, et un intérêt certain dans les années 1980 avec 115 articles ayant une occurrence dont un quart a été retenu. La décennie suivante, avec 124 occurrences, ne nous permet cependant de ne retenir que quinze articles, avant de retomber à quelques articles spécialisés depuis 2001 (figures 3 et 4).

Contrairement aux doctorats, plus de trois quarts des articles de Norois sont consacrés à des espaces européens, et plus de la moitié sur la France. Cela est conforme à la ligne éditoriale de la revue et à la localisation des auteurs, presque tous rattachés à une université ou une institution de l'Ouest français. Dans cet esprit, un tiers des articles se réfère à l'espace atlantique (hors France), sur l'Atlantique nord européen principalement, avec quelques éclairages sur le continent américain oriental et l'Afrique de l'Ouest. En France, les régions de l'Ouest sont les plus étudiées; un tiers des articles sont consacrés à la Bretagne, suivie des autres régions atlantiques, avec une faible occurrence de la Manche (deux articles sur la Normandie). La revue sort peu de sa ligne éditoriale, avec seulement dix articles en 60 ans, cinq donnant une vision mondiale des activités, cinq sur d'autres espaces régionaux (Pacifique et Méditerranée). 


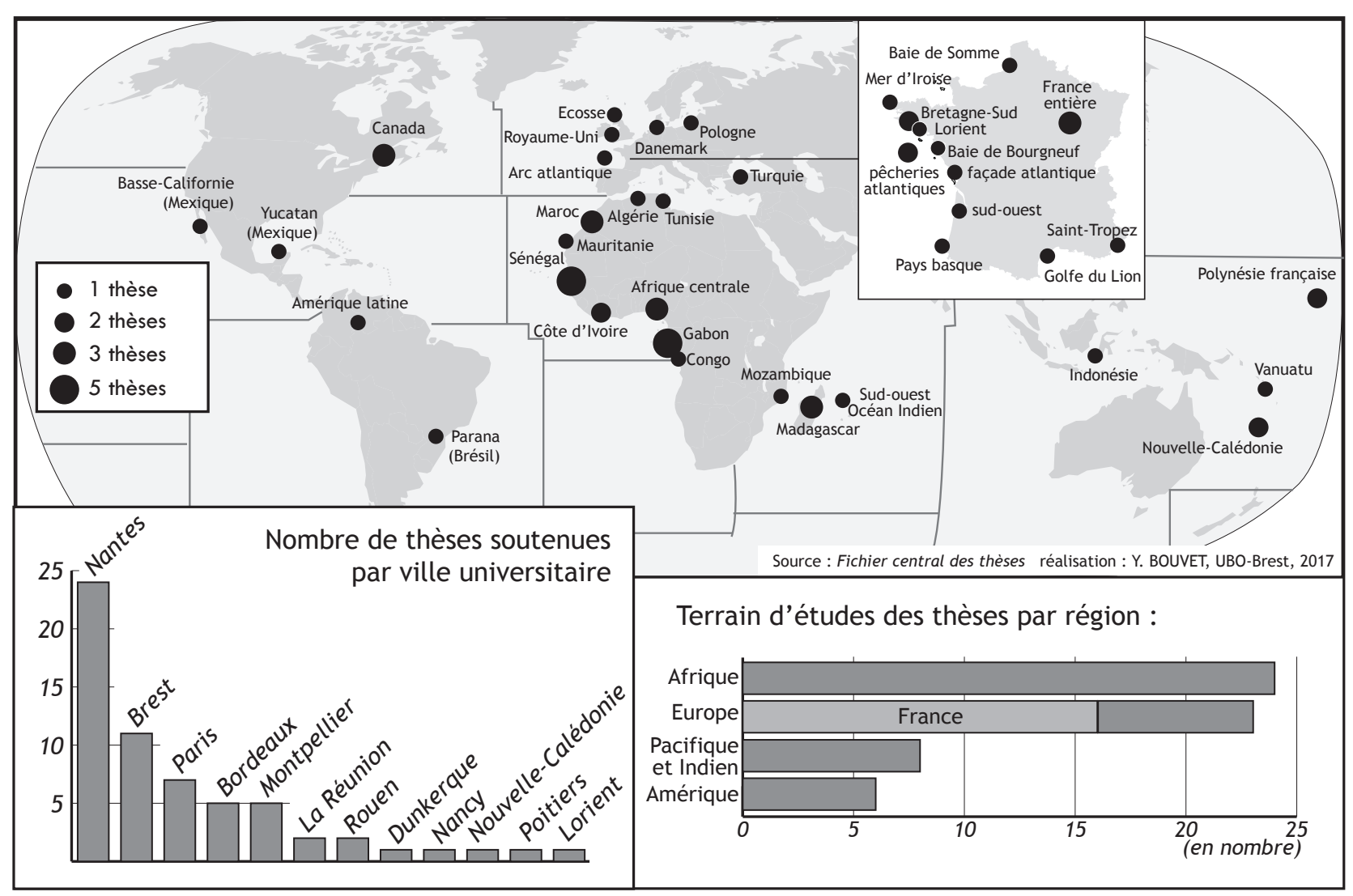

Figure 2 : Les thèses consacrées à la pêche en géographie : espaces étudiés et université d'origine. (source : fichier central des thèses) Doctoral theses in geography devoted to fishing activities: areas studied and candidates' home universities

\begin{tabular}{|c|c|c|c|}
\hline Périodes & $\begin{array}{c}\text { Nombre total } \\
\text { d'articles }\end{array}$ & $\begin{array}{c}\text { Nombre d'articles } \\
\text { retenus }\end{array}$ & $\%$ \\
\hline $1954-1970$ & 4 & 3 & 75 \\
\hline $1971-1980$ & 179 & 24 & 13 \\
\hline $1981-1990$ & 115 & 31 & 27 \\
\hline $1991-2000$ & 124 & 15 & 12 \\
\hline $2001-2017$ & 8 & 2 & 25 \\
\hline Total & 430 & 75 & 17 \\
\hline
\end{tabular}

Tableau 1 : Évolution du nombre d'articles recensés comportant le mot «pêche » dans Norois in Norois

Number of papers reviewed featuring the word "fishing"

\section{Géographie de LA PÊCHe, UN GUIDE ET PLUSIEURS APPROCHES}

Si c'est à André Guilcher, et à Brest, que l'on doit les premières thèses sur la pêche, ce sont ses élèves qui ont bâti les grandes thématiques de recherche en devenant eux-mêmes directeurs de recherche, et donc de thèses.

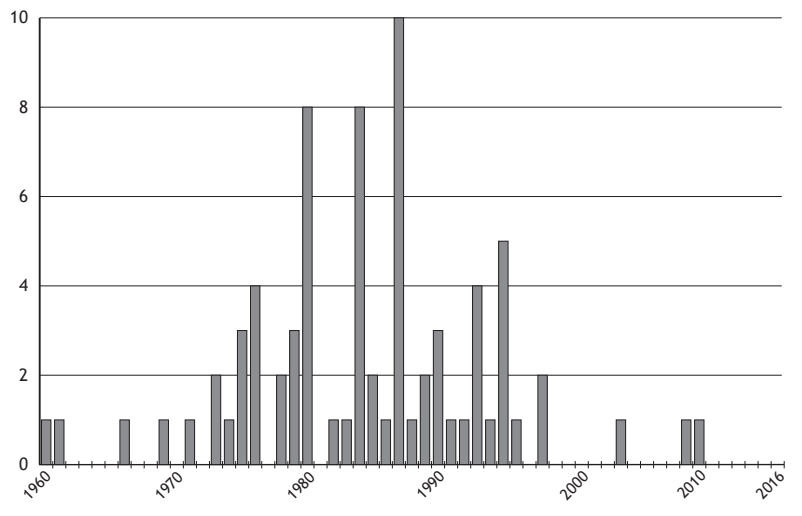

Figure 3 : Nombre d'articles consacrés à la pêche dans Norois par an by year, in Norois

Ainsi, sur 24 thèses réalisées à Nantes, Jean Chaussade-Redon a en encadré douze et JeanPierre Corlay six, leur influence sur les recherches halieutiques est certaine, même si d'autres chercheurs ont contribué à l'émergence de ce thème, notamment chez les tropicalistes, autour de Christian Verlaque à Montpellier, de Jean-Pierre Doumenge 


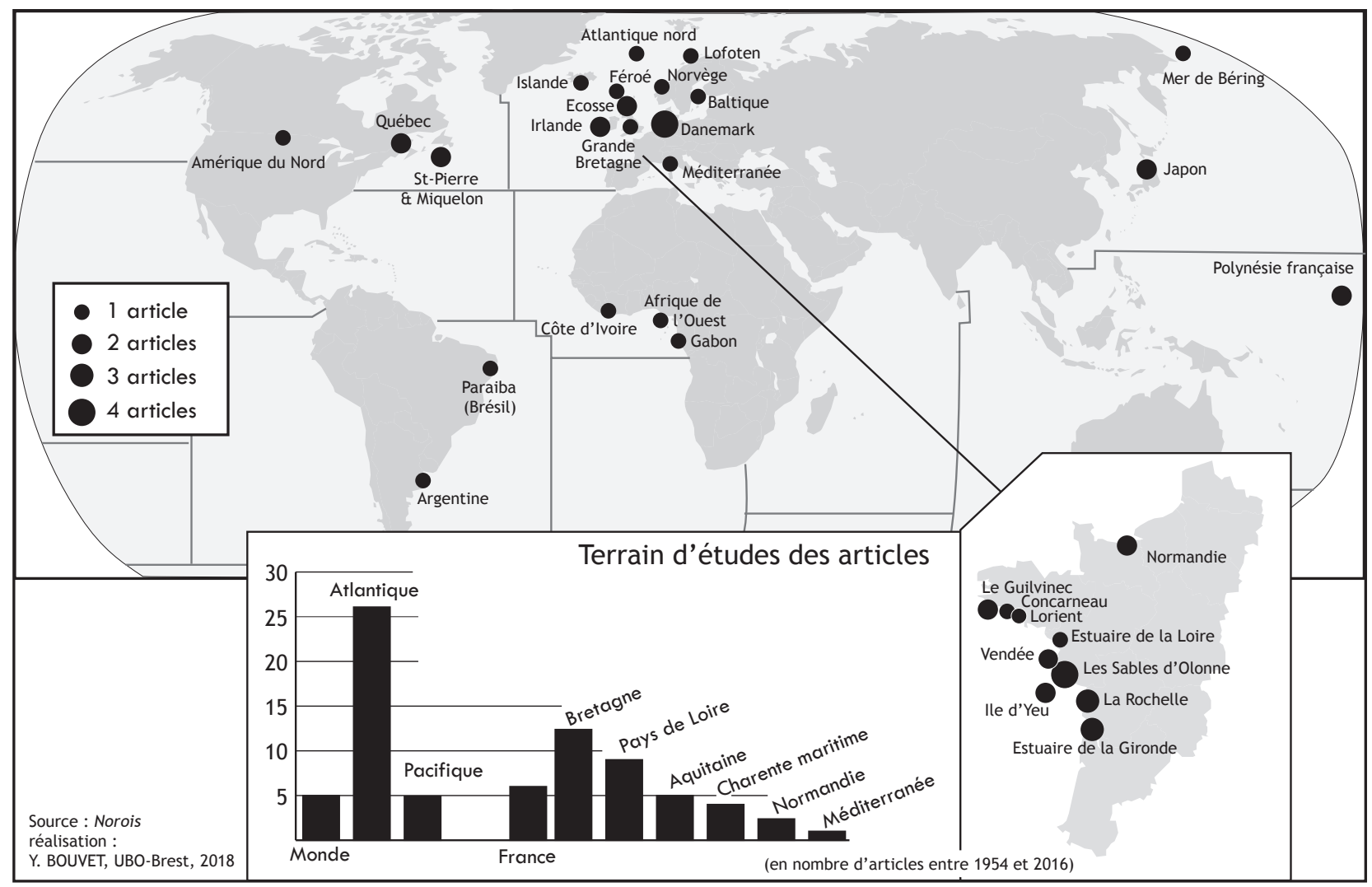

Figure 4 : Les espaces étudiés par les articles centrés sur la pêche en géographie Areas studied in geography papers devoted to fishing

à Bordeaux, ou chez les littoralistes comme JeanClaude Bodéré à Brest. Aujourd'hui, les sujets intégrant la pêche sont plus divers, avec des doctorants attachés à des équipes de recherche ouvertes sur de nombreuses thématiques de la géographie (environnement, géomatique, géopolitique...).

Sur la soixantaine de recherches doctorales, on peut classer les thèses en quatre grands domaines, qui se répartissent sur l'ensemble de la période historique étudiée, même si certains semblent plus présents ces dernières années.

Le premier domaine concerne des études régionales prenant pour axe d'entrée l'activité halieutique, comme élément central d'organisation d'un territoire. Ces études peuvent se faire à l'échelle d'une région (Rieucau, Golfe du Lion, 1994), ou d'une façade (Turan, Mer Noire, 1986; Rakotobe, Arc atlantique, 1998) ou d'un pays (Nadraoui, Maroc, 1991 ; Bignoumba, Gabon, 1995 ; Niang, Sénégal, 2009). On peut y adjoindre les thèses portées vers les espaces maritimes d'un métier, et la mise en avant de la complexité des pêcheries (Pichon, cha- lutiers bigoudens, 1992; Le Rhun, chalutiers pélagiques, 2004). Ces études régionales regroupent un peu moins de deux thèses sur dix réalisées.

Avec plus d'un tiers des thèses présentées, le deuxième domaine va rassembler les chercheurs qui articulent leur recherche autour d'une filière de production (Bouvet, le thon blanc, 1993; ToselloBancal, la sardine, 1994) ou de consommation (Ebolo, Gabon, 1993), autour d'une communauté de pêcheurs (David, Vanuatu, 1991), d'un ensemble portuaire (Couliou, Bretagne méridionale, 1996), mettant à jour l'emboîtement des échelles et les différents espaces construits par la production halieutique. Ils s'inscrivent souvent dans une démarche systémique, construisant peu à peu le géosystème halieutique, autour la thèse fondatrice de JeanPierre Corlay sur le Danemark. La mondialisation, l'organisation européenne des pêches et leurs conséquences sur les filières locales apparaissent dans les travaux les plus récents de ce corpus (Gourlay, 2002 ; Noël, 2011 ; Lembe, 2014). 
Le troisième domaine, avec un quart des travaux doctoraux, va mettre en avant les conséquences du développement des activités halieutiques, notamment les conflits générés par son évolution et les impacts sur les ressources marines (Jagot, provinces atlantiques du Canada, 1990; Grandcolas, Inde du sud, 2006). Ces travaux mettent à jour les conflits potentiels liés à l'expansion d'autres activités littorales ou maritimes (Rieucau, Seine et baie de Somme, 1983; Ekouala, Gabon, 2013), comme le tourisme (Claquin, Mozambique, 2008), la plaisance (Jollit, Nouvelle-Calédonie, 2010), ou plus récemment la mise en place des aires marines protégées (Thomassin, AMP sud-ouest océan Indien, 2011). On peut greffer un quatrième domaine à cette tendance, puisqu'une thèse sur dix s'intéresse à la valorisation du milieu marin par la pêche au côté d'autres activités littorales ou marines, tout en préservant la diversité marine (Aubanel, Moorea, 1993; Beaudin, Golfe du Saint-Laurent, 1997). Depuis le milieu de la décennie 2000, on note aussi une thématique transversale aux thèses relative à la gestion de la ressource et au développement durable (Pioch, récifs artificiels, 2008).

Ces travaux cherchent à montrer la place prise par l'activité halieutique, par la construction d'espaces, de territoires, et avec une approche multiscalaire permettant d'identifier les acteurs et de décrypter l'organisation systémique de ces activités de production. Le jeu des acteurs est aussi de plus en plus au cœur des problématiques, avec une dimension prégnante des sensibilités environnementales et de la préservation des ressources et espaces naturels.

Les thématiques abordées par les articles publiés dans Norois rejoignent celles développées dans les travaux doctoraux, mais elles restent tournées vers des approches régionales. Les auteurs sont en grande majorité des enseignants-chercheurs en géographie, des universités de l'Ouest, et même si des chercheurs d'autres sciences (économie, ethnologie) et d'autres universités (Québec, Afrique, Europe du Nord) apparaissent, ce sont les thématiques de géographie régionale qui émergent le plus dans les articles présentés. Ces universitaires sont aussi porteurs des enseignements en géographie maritime, préparant la relève sur les recherches autour des activités littorales et maritimes. Nous avons choisi de les présenter en fonction du nombre d'articles qu'ils ont réalisé dans Norois, corpus qui est référencé en fin d'article.

Si François Doumenge est le premier à écrire un article sur la filière de consommation en Amérique du Nord en 1961, c'est au cours des années 1970 et 1980 que des universitaires vont fortement publier. Les articles traitent d'études régionales centrées sur l'activité halieutique et principalement sur l'espace Atlantique, en lien avec la ligne éditoriale. On peut suivre et résumer l'évolution analytique et conceptuelle sur ces deux décennies à travers quelques auteurs très présents dans la revue.

Philippe Fournet de l'université de Bordeaux est le plus producteur avec onze articles entre 1975 et 1992. Il s'est intéressé essentiellement à l'espace français atlantique ouest, de Lorient à la Gironde en passant par la Vendée, plus un focus sur les pêches britanniques en crise. Il propose une approche très descriptive de la filière, des métiers, des pêcheurs, des évolutions des métiers et des communautés de pêcheurs.

François Carré de Paris 4-Sorbonne, publie lui six articles, essentiellement sur les mers du nord (Écosse, Béring, Baltique...). Il montre la dépendance à la pêche des territoires périphériques, souvent insulaires, la fragilité des populations et aussi la nécessaire gestion de la ressource pour préserver une société maritime déjà marginale. Bien sûr, il faut ajouter à ces articles, les huit chroniques océanographiques réalisées entre 1994 et 2001 par François Carré qui ont poursuivi le travail d'André Guilcher. Jean-Pierre Corlay, de l'université de Nantes, avec cinq articles, nous propose lui aussi une vision plus sociale et surtout plus systémique de la pêche, intégrant dans ses travaux la place des acteurs sociaux, au-delà des pratiques, des métiers, et mettant à jour les stratégies évolutives, multi-scalaires, décrites à travers des systèmes en interactions (naturels, humains, spatiaux et temporels). Cela fabrique des espaces halieutiques constituant un géosystème. On retrouve chez Jean Chaussade-Redon, directeur de recherches CNRS basé dans une unité mixte de recherche à l'université de Nantes, l'approche de géographie sociale à travers ses cinq articles, approchant les complémentarités des pêches avec les autres activités littorales, tant en Vendée qu'au niveau européen, avec une dimension de changement, de mutation voire de crise que l'on va retrouver dans l'ensemble de son travail de chercheur. 
Ainsi, les études régionales et portuaires dominent les articles en permanence puisque plus de la moitié d'entre eux mettent en avant un port (Gautier, 1966, ports de pêches bretons; Fournet, 1979, Lorient; Bouhier, 1993, Les Sables-d'Olonne), un territoire (Fournet, 1976, Gironde; Chaussade, 1990, île d'Yeu), une région (Corlay, 1976, Danemark; Canevet, 1984, Bretagne). C'est ensuite l'étude d'une filière qui est mis à jour pour plus d'un tiers des articles, souvent associée à un espace particulier (Couliou et Piriou, 1989, pêche thonière; Boude, 1987, pêche artisanale). Les articles consacrés aux mutations (Delbos, 1982, Islande), voire aux crises (Chesnel, 1996, crise pêche rochelaise) et aux conflits (Le Bail, 1998, Atlantique sud-ouest) liés à l'activité halieutique représentent moins d'un article sur cinq, Les plus récents articles éclairent une approche durable de l'activité halieutique dans un contexte de mondialisation des activités (Fleury, Saint-Pierre-et-Miquelon, 2004 ; Noël, 2009, altermondialisation; Bignoumba, 2010, exploitation durable Afrique centrale), reprenant des thématiques émergentes dans les travaux doctoraux. Mais les 20 dernières années sont surtout marquées par une faible production autour de la thématique halieutique, dans un contexte universitaire où la première génération de chercheurs confirmés est partie sans qu'une deuxième ne puisse émerger encore. C'est peut-être la fin de cette thématique particulière en géographie et son intégration dans d'autres approches en géographie ou en sciences humaines et sociales ou de l'environnement.

\section{Approches systémique, globale ET ENVIRONNEMENTALE}

La recherche actuelle en géographie des océans et des littoraux s'appuie sur les approches des pionniers, même si elle privilégie la relation à l'environnement, sa préservation, et l'identification du rôle des acteurs (Miossec, 2001). L'arrivée de la question «Mers et océans » au concours de recrutement des enseignants du secondaire en 2013 a relancé l'édition de manuels et fait apparaître plusieurs auteurs sur la thématique de la gestion des ressources halieutiques (Chadenas et Miossec, 2015; Couliou, 2014; Noël et Koulaï, 2015 ; Trouillet, 2014, 2015; Bouvet, 2014). L'émergence de sujets médiatiques (le thon rouge ou la pêche électrique) peut contribuer à remettre la géographie au cœur des analyses apportant un éclairage sur le rôle de la pêche dans la construction et l'organisation des territoires littoraux, insulaires, marins et terriens. Dans les travaux récents sur la pêche, le renouvellement des ressources et la durabilité des activités halieutiques s'inscrivent dans un contexte de globalisation des filières. La géographie de la pêche voisine avec d'autres sciences humaines et sociales, notamment avec les économistes qui proposent des approches de modélisation et de gestion des pêcheries, des armements, des filières de production mais plus rarement des territoires ${ }^{9}$. Elle voisine aussi avec les sciences de l'environnement, en partageant les mêmes écosystèmes.

C'est ainsi que les publications géographiques sur la pêche se font dans des revues telles Développement durable et territoires, L'espace politique ou VertigO ${ }^{10}$, pour citer les revues en lignes qui émergent depuis les années 2000, avec une approche de gestion intégrée et durable, autour des espaces marins protégés et des problématiques interdisciplinaires, en géopolitique, en géo-économie, en sciences de l'environnement. Les récentes politiques universitaires ont conduit à la mise en place de fédérations de recherche ouvrant des collaborations souhaitées ou contraintes pour obtenir des financements. Si les écoles doctorales thématiques permettent les financements de thèse, encore faut-il convaincre de l'intérêt de faire des travaux sur la pêche. La politique des universités et l'affirmation de thématiques scientifiques des laboratoires se construisent lors des ouvertures de postes, choix souvent difficiles en périodes de restriction budgétaire et de mutualisation des moyens. Aussi, les recherches sur la filière halieutique ne semblentelles pas prioritaires, même dans les universités qui ont vu émerger cette thématique.

9. Tels les chercheurs d'AMURE, Unité mixte de recherche Aménagement des Usages des Ressources et des Espaces marins et littoraux, qui rassemble juristes et économistes en Bretagne occidentale.

10. Revues disponibles en ligne sur la plateforme OpenEdition, revue.org, avec quelques exemples : Wickel A., 2009, Crise du territoire à Mayotte: l'exemple de la pêche artisanale, L'Espace Politique [En ligne], 6 | 20083, URL : [http://journals.openedition.org/espacepolitique/980]. QUEFFELEC B., 2012, Réflexions juridiques sur l'articulation entre biodiversité et planification de l'espace maritime en contexte transfrontalier - illustration franco-belge, Développement durable et territoires [En ligne], vol. 3, $\mathrm{n}^{\circ}$ 3, [http://journals.openedition.org/developpementdurable/9402]. Noël J. et Le Sauce D., 2014, Les pêches artisanales au cœur des systèmes halio-alimentaires durables, VertigO - la revue électronique en sciences de l'environnement [En ligne], Volume 14 Numéro 1, [http:/ journals.openedition.org/vertigo/14901]; DOI : 10.4000/vertigo.14901. 


\section{Conclusion}

Ce regard sur la géographie de la pêche montre l'évolution du cheminement scientifique au gré de l'évolution générale de la géographie, des échanges interdisciplinaires et des évolutions de la société. Les géographes halieutes sont passés d'une approche plutôt vidalienne de monographies régionales, à l'étude de filières économiques structurant des territoires, puis à des approches systémiques, identifiant les espaces, les acteurs et la gouvernance de l'activité halieutique. Les recherches des géographes ont aussi intégré la gestion du milieu marin, à travers la préservation de ses ressources naturelles, sans négliger la pression sociale sur une activité nourricière. L'emboîtement des échelles d'analyse, du local jusqu'au global, est une des lectures qu'apportent les géographes sur le secteur halieutique. Les thèses en cours portent sur la gestion, l'adaptation aux changements, les politiques de gouvernance des espaces marins fragiles, notamment sur les littoraux tropicaux. Elles correspondent aux problématiques posées par la mise en place de politique de gestion des espaces marins et côtiers face aux changements environnementaux. Quant à la publication sur la pêche dans Norois, elle est devenue anecdotique, la gestion des risques côtiers et la transformation des activités littorales y sont les plus représentés, à l'image des travaux des chercheurs œuvrant dans les universités du Grand Ouest. Cette évolution dans la science géographique pourrait être mise en regard des sciences humaines, en élargissant cette recherche bibliométrique afin de cerner la place occupée par la pêche et donc l'intérêt portée à cette activité dans la recherche contemporaine.

\section{AnNeXe I - Corpus d'articles pris en compte par auteur et par ordre de publication \\ (à noter que les fonctions occupées sont indiquées pour les auteurs de deux articles au moins)}

François Doumenge : enseignant-chercheur au MNHN, 1961, Les produits de la mer dans l'économie nordaméricaine, Norois, volume 31, p. 293-319.

1980, Évolution et mutation d'une grande pêche industrielle japonaise : la pêche pélagique des calmars, Norois, volume 106, p. 199-216.

1984, L'aquaculture française : bilan et perspectives, Norois, volume 121, p. 77-96.

1995, L’interface pêche/aquaculture. Coopération, coexistence ou conflit, Norois, volume 165, p. 205222.

Philippe Fournet : enseignant-chercheur à Bordeaux, 1975, L'évolution récente de la pêche Rochelaise, Norois, volume 87, p. 443-461.

1976, La pêche à l'île d'Yeu, Norois, volume 92, p. $555-577$.

1978, Les pêches maritimes charentaises et leurs problèmes, Norois, volume 100, p. 625-630.

1978, Les pêches maritimes britanniques : une activité en crise, Norois, volume 99, p. 319-340.

1979, Lorient, port de pêche industrielle, Norois, volume 102, p. 193-207.

1979, Note sur les activités halieutiques dans l'estuaire de la Gironde, Norois, volume 104, p. 551-554. 1980, Le chalutage pélagique dans les eaux côtières du Sud-Ouest de la France, Norois, volume 106, p. 277-287.

1984, Le dynamisme de la pêche artisanale sablaise, Norois, volume 123, p. 485-495.

1985, La pêche des migrateurs dans l'estuaire de la Gironde : les cycles de l'esturgeon et de la civelle (1920-1985) partie 1, Norois, volume 130, p. 151160.

1986, La pêche des migrateurs dans l'estuaire de la Gironde : les cycles de l'esturgeon et de la civelle (1920-1985) partie 2, Norois, volume 131, p. 335350.

1992, Les marins-pêcheurs de l'estuaire girondin. Approche socio-géographique, Norois, volume 154, p. 121-130.

François Carré : enseignant-chercheur à Paris 4 Sorbonne,

1971, Les paysans-pêcheurs écossais, Norois, volume 71, p. 451-476. 
1973, Quelques aspects des pêches en mer du Nord, Norois, volume 77, p. 59-90.

1975, Les pêches de la Baltique, Norois, volume 88, p. $575-593$.

1980, Les ressources vivantes de la mer de Bering et leur exploitation, Norois, volume 106, p. 157-180. 1990, Insularité et pêche dans les petites îles de l'Atlantique Nord, Norois, volume 145, p. 45-59.

1984, Les grandes pêches thonières françaises en zone tropicale, Norois, volume 121, p. 113-126.

1993, Arrivages de poisson et ports de pêche en Écosse, Norois, volume 160, p. 535-554.

Jean Chaussade-Redon : chercheur CNRS à Nantes, 1973, La pêche artisanale vendéenne et ses problèmes, Norois, volume 78, p. 279-300.

1984, Les difficultés de la pêche industrielle en France : l'exemple de Lorient, Norois, volume 121, p. 141-154.

1990, L'île d'Yeu, un exemple de développement insulaire, Norois, volume 145, p. 81-88.

1993, Perspectives de développement des pêches françaises dans le cadre de l'Arc atlantique, Norois, volume 157, p. 171-176.

1995, Géographie des pêches canadiennes. Un bilan des recherches, Norois, volume 165, p. 173-184.

Jean-Pierre Corlay : enseignant-chercheur à Nantes, 1976, Un milieu original de pêche au Danemark : le Limfjord, Norois, volume 89, p. 87-94.

1976, Un milieu original de pêche au Danemark : le Limfjord (suite), Norois, volume 90, p. 199-220.

1979, La notion d'espace de production halieutique : proposition méthodologique d'étude à partir de l'exemple danois, Norois, volume 4, p. 449-466.

1980, Le poisson industriel au Danemark, Norois, volume 106, p. 251-276.

1984, Le conflit des pêches françaises en 1980 : essai de socio-géographie halieutique, Norois, volume 121, p. 155-169.

Abel Bouhier : enseignant-chercheur à Poitiers, 1993, Le port de pêche des Sables-d'Olonne en 1991, Norois, volume 159, p. 457-460.

1991, Le port de pêche des Sables-d'Olonne au cours de la période 1982-1990, Norois, volume 151, p. 251267.

1993, Le port de pêche et le port de commerce des Sables-d'Olonne (Vendée) en 1992, Norois, volume 160, p. 729-731.

1995, La situation des différents ports de pêche vendéens au début des années quatre-vingt-dix, Norois, volume 167, p. 517-534.
Marcel Gautier : enseignant-chercheur à Rennes, 1960, L'industrie des conserves en Bretagne méridionale (Loire-Atlantique exclue), Norois, volume 27, p. 317-331.

1966, L'évolution récente des principaux ports de pêche bretons, Norois, volume 49, p. 59-71.

Armel Coude : enseignant-chercheur à Caen, 1974, Les Pêches en République d'Irlande. Évolution et structures actuelles, Norois, volume 84, p. 507-526. 1975, Les pêches en République d'Irlande. Production et commercialisation, Norois, volume 85, p. 21-43.

Corentin Canévet : enseignant-chercheur à Rennes, 1980, Les pêches bretonnes : crise et redéploiement, Norois, volume 107, p. 454-456.

1984, L'économie des pêches en Bretagne en 1984, Norois, volume 124, p. 646-650.

André Guilcher : enseignant-chercheur à Brest, 1980, L'exploitation des océans : une présentation, Norois, volume 106, p. 153-156.

1984, Introduction aux grands problèmes maritimes de la France en 1984, Norois, volume 121, p. 5-8.

Geneviève Delbos : chercheure CNRS en ethnologie, 1982, Problèmes humains et économiques de l'Islande, Norois, volume 113, p. 5-16.

1987, Semer du poisson comme on sème du grain... L'aquiculture scientifique et pratique, une histoire vieille d'un siècle..., Norois, volume 133, p. 63-72.

Jean Rieucau : enseignant-chercheur à Lyon, 1984, Mutations halieutiques en Haute-Normandie : de la pêche lointaine au large à une pêche côtière intensive, Norois, volume 121, p. 127-140.

1987, Les pêcheurs des Tiers-Mondes et les enjeux alimentaires nord-sud : de l'indifférence à l'esquisse d'une mobilisation internationale, Norois, volume 133, p. 305-315.

Jean-René Couliou, Nicole Piriou : enseignants-chercheurs à Brest,

1989, Concarneau et la pêche thonière tropicale, Norois, volume 144, p. 357-375.

Nicole Piriou, Jean-René Couliou, 1995, De crise en crise, les pêches de Bretagne méridionale ne sont-elles pas sur la voie de la déstructuration?, Norois, volume 165, p. 185-204.

Jacques Pichon, Nicole Piriou : chercheur et enseignante-chercheure à Brest,

1989, Stratégie d'exploitation des zones de pêche d'un chalutier hauturier du Guilvinec, Norois, volume 141, p. 55-63. 
Joël Le Bail et Claude Nicot : enseignants-chercheurs et enseignant à Brest

1995, Le modèle halieutique bigouden à l'épreuve de la crise, Norois, volume 167, p. 499-515.

Joël Le Bail,

1998, Conflit et coopération dans l'Atlantique du Sud-

Ouest : l'exemple de la gestion des ressources biologiques de la Zone Économique Exclusive argentine, Norois, volume 180, p. 703-714.

Guy-Serge Bignoumba : enseignant-chercheur à Libreville (Gabon),

1998, La pêche atlantique et les États de l'Afrique de l'Ouest, le cas du Gabon, Norois, volume 180, p. 685701.

2010, Les pêches maritimes en Afrique centrale : les préalables à une exploitation durable des ressources, Norois, volume 216, p. 47-56.

Daniel Doeuff,

1969, Les campagnes des pêcheurs bretons dans la région de Dakar : contribution à l'étude des relations entre le milieu océanographique et les lieux de pêche, Norois, volume 64, p. 567-583.

Jacques Pinard,

1976, Les industries dérivées de la pêche en Norvège, Norois, volume 90, p. 177-198.

Jean-Pierre Pinot, 1980, L'affectation des fonds de pêche à des groupements d'exploitation en commun (nord du golfe de Gascogne), Norois, volume 106, p. 289-300.

Jean Domingo,

1980, Aspects de l'évolution récente des pêches artisanales en Côte-d'Ivoire, Norois, volume 106, p. 181198.

Jean-François Abgrall, Pierre Rainelli, 1983, La pêche québécoise et son contexte général, Norois, volume 119, p. 333-347.

Alistair Cruickshank, 1985, The Lofoten spawning cod fishery, La pêche à la morue frayante des îles Lofoten, Norois, volume 127, p. $413-428$

Denis Bailly,

1987, Quelques réflexions sur la conception japonaise de l'aménagement des pêches, Norois, volume 133, p. 275-287.

J.-L. Bascoul , J.-P. Brouat, 1987, Le rapport des marins-pêcheurs aux plaisanciers : un analyseur de leurs représentations de la mer comme espace professionnel, Norois, volume 133, p. 297-303.
Gildas Borel, 1987, Pêche artisanale et construction navale en Polynésie française, Norois, volume 133, p. 347-353.

Jean-Pierre Boude, 1987, Effets économiques induits par la pêche artisanale : méthodologie et résultats appliqués à la BasseNormandie, Norois, volume 133, p. 289-296.

Laurence Caillaud, 1987, Stratégie de pêche et parcs à poissons dans un atoll de Polynésie française, Norois, volume 133, p. 331-346.

Gilbert David, 1987, Évolutions technologique et sociale de la pêche artisanale sur le littoral de l'état de la Paraiba (nord-est du Brésil), Norois, volume 133, p. 317-330.

J.-L. Durand et S. Girard, 1987, Schémas d'évolution des activités de pêche, Norois, volume 133, p. 391-405.

François Feral,

1987, Un hiatus dans l'administration et la politique des pêches maritimes : les prud'homies de pêcheurs en Méditerranée, Norois, volume 133, p. 355-369.

Jocelyne Marchand,

1987, Rôles de l'estuaire de la Loire vis-à-vis des espèces d'intérêt halieutique, Norois, volume 133, p. 379-390.

André-Louis Sanguin, 1988, Un litige franco-canadien actuel : la zone économique des 200 milles à Saint-Pierre-et-Miquelon , Norois, volume 137, p. 85-89.

B. Raoulx, 1990, Autonomie politique et changement social dans une société halieutique : le cas des îles Féroé, Norois, volume 146, p. 131-146.

Roger Renard, 1994, Le transfert du port de pêche de La Rochelle à Chef-de-Baie, Norois, volume 164, p. 725-733.

Marc Chesnel, 1996, La crise de la pêche rochelaise à l'épreuve d'une stratégie portuaire mal calibrée, Norois, volume 172, p. 775-789.

Christian Fleury, 2004, Saint-Pierre et Miquelon, îles frontière, Norois, volume 190, p. 25-40.

Julien Noël, 2009, Regard géographique sur les dimensions spatiales de l'altermondialisation halieutique, Norois, $n^{\circ} 211$, p. 7-21. 


\section{Annexe 2 - Travaux de thèses cités par ordre chronologique}

Chaussade-Redon Jean, 1980. La pêche et les pêcheurs des provinces maritimes $d u$ Canada. Thèse de doctorat d'État en Géographie, Université de Brest, 302 p.

Rieucau Jean, 1983. La pêche de l'estuaire de la Seine à la Baie de Somme et les occupations conflictuelles du littoral. Thèse $3^{\mathrm{e}}$ cycle en géographie, Université de Brest, $386 \mathrm{p}$.

Turan Fuat, 1986. La pêche sur le littoral turc de la Mer Noire. Thèse de doctorat en géographie, Université de Montpellier 3, $876 \mathrm{p}$.

Carré François, 1988. Pêches maritimes et pêcheries de l'Écosse. Thèse de doctorat d'État en Géographie, Université de Brest, 5 volumes, $1870 \mathrm{p}$.

JAGOT Loïk, 1990. Coopération maritime et développement régional dans les provinces atlantiques du Canada. Thèse de doctorat en géographie, Université de Nantes, 550 p.

David Gilbert, 1991. Pêche villageoise et alimentation au Vanuatu : exploration d'un système. Thèse de doctorat d'État en Géographie, Université de Brest, 1052 p.

Nadraoui Mustapha, 1991. La pêche maritime sur la façade atlantique du Maroc. Thèse de doctorat en géographie, Université de Nancy 2, 355 p.

Pichon Jacques, 1992. Les zones de pêche des chalutiers bigoudens, Thèse de doctorat en géographie, Université de Brest, 298 p.

Aubanel Annie, 1993. Valeurs socio-économiques du milieu corallien récifal et de ses ressources : application à une île océanique du Pacifique sud: Moorea, archipel de la Société. Thèse de doctorat en géographie, Université de Bordeaux 3, 313 p.

Bouvet Yvanne, 1993. La Pêche du thon blanc en France: Aspects géographiques d'une activité maritime saisonnière. Thèse de doctorat en géographie, Université de Nantes, $322 \mathrm{p}$.

Corlay Jean-Pierre, 1993. La pêche au Danemark, essai de géographie halieutique, Thèse de doctorat d'État en Géographie, Université de Brest, 1331 p.

Eвolo Clet Mesmin Edou, 1993. Marché du poisson au Gabon. Thèse de doctorat en géographie, Université de Montpellier 3, $398 \mathrm{p}$.

Rieucau Jean, 1994. Des sociétés maritimes et riveraines de la Méditerranée française: l'exemple du Golfe du Lion. Thèse de doctorat en géographie, Université de Paris 4, 675 p.

Tosello-Bancal François, 1994. L'évolution de la pêche de la sardine sur le littoral français. Thèse de doctorat en géographie, Université de Paris 4, 216 p.

Bignoumba Guy Serge, 1995. La pêche maritime au Gabon: contribution à l'étude géographique d'une activité secondaire dans un pays tourne principalement vers l'exploitation de ses ressources continentales. Thèse de doctorat en géographie, Université de Nantes, 372 p.

Couliou Jean-René, 1996. Les ports de pêche hauturière de Bretagne méridionale : étude géographique de la mutation d'un système halieutique. Thèse de doctorat en géographie, Université de Brest, 628 p.
Beaudin Maurice, 1997. L'adaptation économique des zones maritimes de pêche : le cas des communautés du golfe $d u$ Saint-Laurent. Thèse de doctorat en géographie de l'université de Nantes, 675 p.

Raкотове Suze R., 1998. Pêche et aquaculture dans les régions de l'arc atlantique (ou comment des activités de type primaire peuvent-elles contribuer à la régénération de régions marginales?). Thèse de doctorat en géographie, Université de Nantes, 387 p.

Gourlay Florence, 2002. La mondialisation à l'échelle d'une ville moyenne maritime : Le cas de Lorient. Thèse de doctorat en géographie, Université de Lorient, 536 p.

Le Rhun Philippe, 2004. Le chalutage pélagique dans les eaux territoriales bretonnes et vendéennes. Thèse de doctorat en géographie, Université de Brest, 435 p.

Grandcolas Delphine, 2006. Pêcheurs, État et mondialisation: stratégies des populations locales et gestion des ressources naturelles : le cas du delta de la Godavari, Inde du sud. Thèse de doctorat en géographie, Université de Paris, 316 p.

Claquin Bérengère, 2008. Approche culturelle des communautés de pêcheurs traditionnels et mise en tourisme des îles $d u$ Mozambique : étude de cas des archipels de Bazaruto et des Quirimbas. Thèse de doctorat en géographie, Université de La Réunion, 269 p.

Pioch Sylvain, 2008. Les « habitats artificiels » : élément de stratégie pour une gestion intégrée des zones côtières? : essai de méthodologie d'aménagement en récifs artificiels adaptés à la pêche artisanale côtière. Thèse de doctorat de l'université Paul-Valéry, Montpellier, France et Tokyo University Marine science, Tokyo, Japon, 288 p.

Ndeye Astou N., 2009. Dynamique socio-environnementale et développement local des régions côtières du Sénégal: l'exemple de la pêche artisanale. Thèse de doctorat en géographie, Université de Rouen, 301 p.

NoËL Julien, 2011. Regards géographiques sur la mondialisation halieutique. L'altermondialisation et les formes de résistances des «pêches artisanales ». Thèse de doctorat en géographie, Université de Nantes, 484 p.

Jollit Isabelle, 2010. Spatialisation des activités humaines et aide à la décision pour une gestion durable des écosystèmes coralliens : la pêche plaisancière dans le lagon sud-ouest de la Nouvelle-Calédonie. Thèse de doctorat en géographie, Université de la Nouvelle-Calédonie, 558 p.

Ekouala Landry, 2013. Le développement durable et le secteur des pêches et de l'aquaculture au Gabon : une étude de la gestion durable des ressources halieutiques et leur écosystème dans les provinces de l'Estuaire et de l'Ogooué Maritime. Thèse de doctorat en géographie, Université de Dunkerque, 368 p.

Lembe Aline-Joëlle, 2014. Pêches maritimes et développement durable dans les états côtiers d'Afrique centrale : des dysfonctionnements à l'exploitation durable des ressources halieutiques. Thèse de doctorat en géographie, Université de Nantes, 401 p. 


\section{Bibliographie}

Besançon J., 1965. Géographie de la pêche, Paris, Gallimard, $523 \mathrm{p}$.

Bodéré J.C., Carré F., Moign A., Simon T., 1984. Chroniques arctiques, Histoire des recherches et de la mise en valeur, une géographie de l'Arctique et de l'Antarctique, Norois, $n^{\circ} 122$, p. 299-332.

Bouvet Y., 2014. «De la mer à l'assiette : présentation de la filière halieutique dans le Monde », Géoconfluences, [http:// geoconfluences.ens-lyon.fr/informations-scientifiques/dossiers-thematiques/oceans-et-mondialisation/corpus-documentaire/de-la-mer-a-12019assiette-presentation-de-lafiliere-halieutique-dans-le-monde].

Carré F., 2009. Les géographes et la connaissance scientifique de la mer, une approche épistémologique sur le travail des géographes, in Actes du FIG 2009, [http://archives-fig-stdie.cndp.fr/actes/actes_2009/carre/article_1.html].

Carré F., 1995. André Guilcher (1913-1993), une vie de géographe. (1 photo, 1 fig.). Bibliographie des travaux d'André Guilcher de 1982 à 1994. Norois, nº 165, p. 7-30.

Chadenas C., Miossec A., 2015. Acteurs et territoires de la protection des espaces remarquables sur les océans : de la sanctuarisation au développement durable, in GuilLaume J., Espaces maritimes et territoires marins, Paris, Ellipses, p. 147-163.

Chaussade J., 1994. La mer nourricière: enjeu du XXI siècle, Champtoceaux, $153 \mathrm{p}$.

Corlay J.-P., 1995. Géographie sociale, géographie du littoral, Norois, Nantes, no 165, p. 217-265.

Corlay J.-P., 1993. L'espace halieutique existe, je l'ai rencontré... essai théorique et méthodologique sur la géographie de la pêche, Cahiers nantais, no 40, p. 57-75.
Couliou J.-R., 2014. Les ressources vivantes de la mer, in Moissec A., Géographie des mers et océans, Rennes, PUR, p. 103-148.

Deboudt P., 2011 . Le littoral et la mer dans les revues Hommes et Terres du Nord et Territoire en Mouvement (1963-2007), Géocarrefour, Vol. 86/3-4, 15 p., [http://geocarrefour.revues. $\operatorname{org} / 8435]$

Doumenge F., 1965. Géographie des mers, Paris, PUF, coll. « Magellan », 279 p.

Goeldner-Gianella, L., 2012. Du littoral à la haute mer : quelles recherches récentes en géographie?, EchoGéo, $\mathrm{n}^{\circ} 19,6 \mathrm{p}$.

Miossec A., 2001. L'évolution de la géographie des océans et des littoraux face aux perspectives du développement durable au $\mathrm{xxI}^{\mathrm{e}}$ siècle, quelles hypothèse envisager?, Annales de géographie, Paris. p. 509-526.

Nö̈L J., Koulaï E. . 2015, Les dynamiques spatiales des activités halieutiques, in Escach N., Géographie des mers et des océans, Paris, Dunod, p. 155-181.

Nö̈L J., 2013. « Le système-monde aquatique : panorama géographique d'une filière globalisée ", L'information géographique, vol. 77, n 1, p. 76-100.

Regrain R., 1995. André Guilcher, géographe des littoraux : une œuvre en son contexte. Norois, $n^{\circ} 165,1995$, p. 31-35.

Trouillet B., 2014. Ressources vivantes de la mer, in Deboudt J. et al., Géographie des mers et des océans, Paris, Armand Colin, p. 115-140.

Troulllet B., 2015. Les enjeux spatiaux : la reconfiguration des espaces halieutiques, in Guillaume J., Espaces maritimes et territoires marins, Paris, Ellipses, p. 53-88. 